\title{
Design and development of a new dedicated RF sensor for the MRI of rat brain
}

\author{
Aktham Asfour
}

Joseph Fourier University of Grenoble, Grenoble, France.

Email: Aktham.Asfour@uif-grenoble.fr

Received 14 October 2009; revised 13 November 2009; accepted 16 November 2009.

\begin{abstract}
The design and development of a new dual-frequency RF probe-head are presented. This probe was initially dedicated for the MRI of both proton $\left({ }^{1} \mathrm{H}\right)$ and hyperpolarized Xenon-129 (HP $\left.{ }^{129} \mathrm{Xe}\right)$ in the rat brain at 2.35 Tesla. It consists of a double-tuned $(100 \mathrm{MHz}-$ 27.7 MHz) volume coil, which could be used for both transmitting and receiving, and of a receive-only single-tuned (27.7 MHz) coil. The double-tuned coil consists of two concentric birdcage resonators. The inner one is a low-pass design and it is tuned to $27.7 \mathrm{MHz}$, while the outer one, tuned to $100 \mathrm{MHz}$, is high-pass. The receive-only coil is a surface coil which is decoupled from the double-tuned volume coil by an active decoupling circuitry based on the use of PIN diodes. A home-built Transmit/Receive (T/R) driver ensures biasing of the PIN diodes in both volume and surface coils. The original concepts of the design are addressed, and practical details of realization are presented. One of the underlying ideas behind this work is to proceed well beyond the application to the MRI of HP ${ }^{129} \mathrm{Xe}$. Actually, this design could be easily adapted for a large palette of other MRI applications. Indeed, we tried to make the design versatile, simple and easy to replicate by other research groups, with a low-cost, minimum development time and accepted performances. The prototype was validated at 100 $\mathrm{MHz}$ and at 26.4 MHz (sodium-23 resonance frequency at 2.35 T). MRI experiments were performed using phantoms. In vivo ${ }^{1} \mathrm{H}$ images and ${ }^{23} \mathrm{Na}$ spectra of the rat brain are also presented.
\end{abstract}

Keywords: MRI; RF Coils; Double-Tune; Active Decoupling; Proton; Sodium-23; Hyperpolarized Xenon-129

\section{INTRODUCTION}

Nuclear Magnetic Resonance (NMR) and Magnetic

${ }^{1}$ In healthy brain of adult humans, the $\mathrm{CBF}$ is regulated to an average of 50 milliliters of blood per 100 grams of brain tissue per minute.

${ }^{2}$ Value of the $\mathrm{CBF}$ measured over all the brain.

${ }^{3}$ Value of the $\mathrm{CBF}$ measured within a specific brain region.
Resonance Imaging (MRI) are important diagnostic and analytical tools for biomedical studies. During the last two decades, and since the MRI of hyperpolarized xenon-129 (HP $\left.{ }^{129} \mathrm{Xe}\right)$ was initially demonstrated in 1994 [1] in mouse lungs, the MRI of hyperpolarized gases $\left({ }^{129} \mathrm{Xe},{ }^{3} \mathrm{He} \ldots\right)$ have become widespread for a large palette of applications in biology and medicine. Particularly, the MRI of injected or inhaled $\mathrm{HP}{ }^{129} \mathrm{Xe}$ is a promising noninvasive method for the investigation of brain function and clinical use. In fact, several studies carried out in our laboratory and by some research groups in the world, have already demonstrated that the MRI of HP

${ }^{129} \mathrm{Xe}$ may allow quantitative measurement of the cerebral blood flow (CBF) with a high spatial resolution $[2,3,4,5]$. This physiological parameter, which is defined as the blood supply to the brain in a given time and per mass unit of brain tissue ${ }^{1}$, is tightly regulated to meet the brain's metabolic demands. The measurement of changes in both global cerebral blood flow $(\mathrm{gCBF})^{2}$ and regional cerebral blood flow $(r C B F)^{3}$ is of great value for functional brain studies as well as for the diagnosis of a large number of brain diseases (Alzheimer, epilepsy, Parkinson, ischemia...). Nevertheless, for such measurements, especially those of the $r C B F$, to become quantitatively reliable by the MRI of $\mathrm{HP}{ }^{129} \mathrm{Xe}$, one must first overcome several experimental and instrumental challenges.

In addition to the understanding of the behavior of the ${ }^{129} \mathrm{Xe}$ in the brain, which is out of the scope of this paper, many instrumental obstacles have to be actually resolved. For example, the experimental set-up for polarization build-up and storage is relatively complicated and require some know-how. One important aspect in building this polarization set-up has already been addressed in our previous works [6,7]. A second instrumental challenge concerns the development of specific radiofrequency (RF) probes for these experiments. In this paper, we deal with this problem and we present an original and versatile design of a dedicated RF double-tuned volume coil actively-decoupled from a simple-tuned receive-only RF surface coil.

To help the reader in better understanding the general context of our research and the goal of this paper, one may 
need to review or to learn some basic principles of a typical NMR experiment as well as of the NMR of $\mathrm{HP}^{129} \mathrm{Xe}$.

As it is well known, when a sample, consisting of NMR-sensitive nuclei $\left({ }^{1} \mathrm{H},{ }^{3} \mathrm{He},{ }^{129} \mathrm{Xe} ;{ }^{23} \mathrm{Na}\right.$...etc. $)$, is subjected to a uniform static magnetic field $B_{0}$, a net macroscopic magnetization $M$ of the sample appears in the direction of $B_{0}$. This magnetization is proportional, roughly speaking, to the polarizing field $B_{0}$, to the density of the nuclei within the sample and to the characteristic gyro-magnetic ratio $\gamma$ of the nucleus being studied.

If the sample is subjected now to a short pulse (called excitation pulse) of a second radiofrequency magnetic field $\mathrm{B}_{1}$, applied at the characteristic Larmor frequency $f_{0}$ (about $100 \mathrm{MHz}$ for ${ }^{1} \mathrm{H}, 27.7 \mathrm{MHz}$ for ${ }^{129} \mathrm{Xe}$ and 26.4 $\mathrm{MHz}$ for ${ }^{23} \mathrm{Na}$ at $B_{0}=2.35$ Tesla), the net magnetization, $M$, may be "tipped" or rotated from its initial direction (or from its equilibrium state) by an angle his angle is called "flip angle". It is proportional to $\mathrm{B}_{1}$ according to the equation $\alpha=\gamma \cdot \tau \cdot B_{1}$ where the duration of the RF pulse is. At the end of the excitation pulse, a resonance signal (or the NMR signal) at the same frequency, $f_{0}$, is received. This signal, which is proportional to the magnetization $M$ and to $\gamma$, is processed to be used for obtaining a "fingerprint" of the environment of the nucleus being studied.

At equilibrium and for a same magnetic field $B_{0}$, the NMR signal of a given ${ }^{129} \mathrm{Xe}$ population is about 10000 times lower than the one that could be obtained from the same volume of protons. This is because of the intrinsic lower gyro-magnetic ratio and lower density of the xenon. Consequently, at equilibrium, the in vivo-NMR signal of the injected or inhaled ${ }^{129} \mathrm{Xe}$ is not exploitable. To compensate this limitation, hyperpolarizing techniques have been successfully used to dramatically increase the magnetization of ${ }^{129} \mathrm{Xe}$ before using it for the in vivo experiments. In this case, the magnetization and the NMR signal levels are typically enhanced by about five orders of magnitude.

In NMR/MRI experiments, the well-tuned (to the frequency $f_{0}$ ) RF probe has usually a dual role: it ensures the excitation of the sample (i.e., it creates the RF field $B_{1}$ ) and it receives the weak NMR signal from this same sample. Usually, both excitation (transmission) and receiving processes are produced in the same coil. Nevertheless, there are cases where these processes are produced by two or more different coils. Generally, according to considerations related to the spatial homogeneity of the excitation field $\mathrm{B}_{1}$ and to the receiving sensitivity, RF coils may be categorized, roughly speaking, as either volume coils or surface coils. Volume coils create homogeneous spatial distribution of $\mathrm{B}_{1}$ and guarantee the possibility to image deep organs. Surface coils allow high sensitivity, and hence high signal-to-noise ratio (SNR), for surface regions (the rat brain could be considered as surface region). These surface coils present however a poor $\mathrm{B}_{1}$ homogeneity when compared with volume coils. This homogeneity decreases generally when one moves away for the centre of the coil.

Both volume and surface coils could be used for both transmission and receiving. However, when $\mathrm{B}_{1}$ homogeneity during the transmission period is required, the use of a transmit volume coil is necessary. On the other hand, when this homogeneity is not an issue, a surface coil may be preferred to optimize the SNR of the detected NMR signals. The association of a volume coil, for transmission, decoupled from a surface coil, for receiving, is usually used when both $B_{1}$ uniformity and high SNR are required.

For our experiments of measuring the $r C B F$ by the NMR of $\mathrm{HP}{ }^{129} \mathrm{Xe}$, a dedicated RF probe has to be built. Actually, the measurement method of the gCBF requires knowledge of the global (over all the rat brain) flip angle $[2,3,4,5]$. This angle depends, remember, on $B_{1}$. The determination of a global flip angle can be easily performed. However, when local or regional cerebral blood flow $(r C B F)$ values (within a specific brain region or inside a pixel of the MRI image) have to be measured, the local flip angles (which depend on the local values of $\mathrm{B}_{1}$ ) have to be accurately estimated. This estimation is not a trivial task. This is why it's generally assumed that local flip angles are close enough to the global value. Nevertheless, when a surface coil is used for transmission, the estimated global flip angle could be very different from the local values due the inherent non-uniformity of the $\mathrm{B}_{1}$ field distribution. This may produce inaccurate results in the $r C B F$ values. For example, Venkatesh et al. have noted a $20 \%$ difference between the decay times (parameter directly related to the $r C B F$ ) obtained with a surface coil and those obtained with a volume coil used for both transmission and receiving [3]. Moreover, since Kilian et al. [2] used a surface coil in their human brain measurements, it is possible that their results contain a systematic error due to inaccurate estimation of the local flip angles. The same problem was also encountered in our experiments [5]. In all these experiments, the use of surface coils was initially motivated by the desire to obtain a high SNR in the MRI images.

To overcome this problem, the idea we develop here consists in using a volume coil for transmitting so as the global flip angle will be close enough to the local ones thanks to the high $\mathrm{B}_{1}$ homogeneity guaranteed by this type of coils. The detection of the NMR signals will then be performed by a surface coil positioned inside the volume coil. In this situation the advantage the inherent high sensitivity associated with the surface coil is retained.

The two coils must be decoupled so as when the first is active, the second must be deactivated. In other words, during transmission, the volume coil is active or "on" while the surface coil is "off". During receiving, the transmit volume coil has to be deactivated and the sur- 
face one has to be active. Both transmit and receive coils must be well-tuned to the resonance frequency of ${ }^{129} \mathrm{Xe}$ (27.7 MHz at 2.35 T).

Moreover, for the $r C B F$ measurements by MRI, the final "ideal" probe must satisfy more stringent requirements. Actually, in addition to the transmission and receiving at the frequency of ${ }^{129} \mathrm{Xe}$, one has to be able to perform ${ }^{1} \mathrm{H}$-MRI experiments (at $100 \mathrm{MHz}$ ) without changing the probe and without moving the sample under test (rat in our application). In fact, acquiring ${ }^{1} \mathrm{H}$ images is necessary to localize anatomical structures in the rat brain. These anatomical images are to be superposed to ${ }^{129} \mathrm{Xe}$ images to identify structures where inhaled or injected ${ }^{129} \mathrm{Xe}$ could be localized in the rat brain. On the other hand, the procedure of homogenization of the static magnetic field (shimming), which is essential before every MRI acquisition, must be performed using ${ }^{1} \mathrm{H}$ NMR signals. Actually, since this shimming procedure requires repetitive excitations and detections, it can not be accomplished on the ${ }^{129} \mathrm{Xe}$ signals because the artificial magnetization (non equilibrium state) of the $\mathrm{HP}{ }^{129} \mathrm{Xe}$ is not renewable when destroyed by an RF excitation pulse.

In both anatomical image acquisition and shimming procedures, a high SNR of the detected ${ }^{1} \mathrm{H}$ signals is not an issue and a same transmit-receive volume coil tuned to $100 \mathrm{MHz}$ could be adequate. This coil must however be decoupled from both transmit volume and receiveonly ${ }^{129} \mathrm{Xe}$ coils.

To summarize: the final "ideal" NMR probe that we developed consists of a transmit volume coil actively decoupled from a receive-only surface coil working at 27.7 MHz, and a transmit/receive volume coil for the ${ }^{1} \mathrm{H}$ MRI at $100 \mathrm{MHz}$. The decoupling between the ${ }^{1} \mathrm{H}$ coil and the ${ }^{129} \mathrm{Xe}$ coils will be performed geometrically.

This novel configuration, which actually associates three MRI coils in a same probe, is new and has not been published. In fact, due to the variety of size and experimental protocols encountered with small-animal imaging experiments, it is usually advisable and may be necessary to build a probe optimized for each experimental set-up and for each application. However, it is important to notice here that another objective of this work is to proceed well beyond this specific application to the MRI of HP ${ }^{129} \mathrm{Xe}$. Actually, the current design could be adapted or could inspire other designs for a palette of MRI applications in the rat brain. Generally, for such applications, the "ideal" imaging/spectroscopy coil would be one which provides high sensitivity, high $\mathrm{B}_{1}$ homogeneity, and operation on two or more frequencies without retuning requirement or cables changes. The problem of sensitivity can be addressed using surface coils [8]. The homogeneity requirements can be met by the use of adequate coil geometry (i.e., birdcage design [9]). The problem of acquiring data from two different nuclei (usually proton and a second nucleus like ${ }^{129} \mathrm{Xe}$, ${ }^{23} \mathrm{Na}$ sodium, ${ }^{31} \mathrm{P}$...) requires the use of an efficient dou- ble-tuned coil (volume or surface coils) $[10,11,12,13,14$, $15,16,17]$. While these problems have been addressed individually, there have been no published designs that have integrated all these features into one design. The goal of this work is to approach performances as outlined above. Another underlying idea is to make this specific probe easy to replicate, by other research groups with a low-cost, minimum development time and accepted performances. To allow for easy extension of this design to other frequencies (nuclei) and dimensions, we describe in some details the practical aspects of the workbench design as well as the coil characterization and MRI/NMR testing using phantoms. The probe is validated at the ${ }^{1} \mathrm{H}$ frequency and the sodium frequency $(26.4 \mathrm{MHz}$ at $2.35 \mathrm{~T})$ since this last is very close to the xenon frequency. In vivo MRI images of the rat brain were obtained at ${ }^{1} \mathrm{H}$ frequency as well as NMR signals at the sodium frequency.

All developed circuits and any other details on developed probe could be obtained by simply writing to authors.

\section{MATERIALS AND METHODS}

\subsection{The Double-Tuned Volume Probe}

We built a double-tuned volume probe using the birdcage design [9]. This design ensures inherent high $\mathrm{B}_{1}$ homogeneity within the coil region. It is therefore well-suited for the MRI of HP ${ }^{129} \mathrm{Xe}$. The design is shown in its low-pass and high-pass versions in Figure 1. It consists of two circular loops, referred to as end-rings, connected by a number of equally spaced straight segments referred to as legs or rungs. Depending on the version, the tuning capacitors are placed in the legs (low-pass) or in the end-rings (high-pass).

A single-tuned birdcage will resonate in several modes. A birdcage with $\mathrm{N}$ legs has $\mathrm{N} / 2$ resonance modes (assuming $\mathrm{N}$ is even) $[9,17]$, with the most useful to MRI being Mode 1 (fundamental mode). In this mode, the currents in the legs follow a cosine distribution as one moves radially around the coil. This sinusoidal distribution of currents is essential to create a very homogenous transverse magnetic field $B_{1}$.

For our application, it is necessary to double-tune the birdcage. The sinusoidal optimum current distribution should be maintained at each frequency. Doubletuning a birdcage resonator is not a trivial task and it has been subject of many publications $[10,11,12,13,17]$. The method to choose depends actually on the desired performances at each frequency. Double-tuned birdcages have been used at relatively low field $(\leq 1.5 \mathrm{~T})$. Few designs at higher fields have been proposed. For example, Fitzsimmons et al. [10] presented a double-tuned birdcage coil at $2 \mathrm{~T}$. It was dedicated for the MRI of proton $(85.5 \mathrm{MHz})$ and the NMR spectroscopy of phos- 
phorus (34.6 MHz) of the rat abdomen. They derived their coil in quadrature operation for both proton and phosphorus channels. The coil was used for both transmission and receiving at each frequency. One of the originalities of the double-tuned birdcage that we propose here is that it has to work at higher field $(2.35 \mathrm{~T})$ for the MRI of the rat brain. More important is the fact that, to the knowledge of the author, a double-tuned birdcage associated with an actively decoupled receiveonly surface coil has not been published.

The double-tuned birdcage we built consists of two birdcages in a concentric configuration as it is seen in Figure 2.
Each resonator consists of eight legs. This number represents a compromise between $\mathrm{B}_{1}$ homogeneity and complexity of realization. The inner coil, a low-pass birdcage, had a diameter of $9 \mathrm{~cm}$, a length of $13 \mathrm{~cm}$, and was tuned to the xenon frequency. The outer coil, a high-pass design, had a length of $16 \mathrm{~cm}$, a diameter of $10.5 \mathrm{~cm}$, and was tuned to the proton frequency. We verified that no proton resonance modes occur at the xenon frequency. Conversely, no xenon modes occur at the proton frequency. The inner birdcage was rotated within the outer birdcage until minimum coupling occurred. This positioned the legs of the inner coil midway between the legs of the outer one. Also, the outer bird

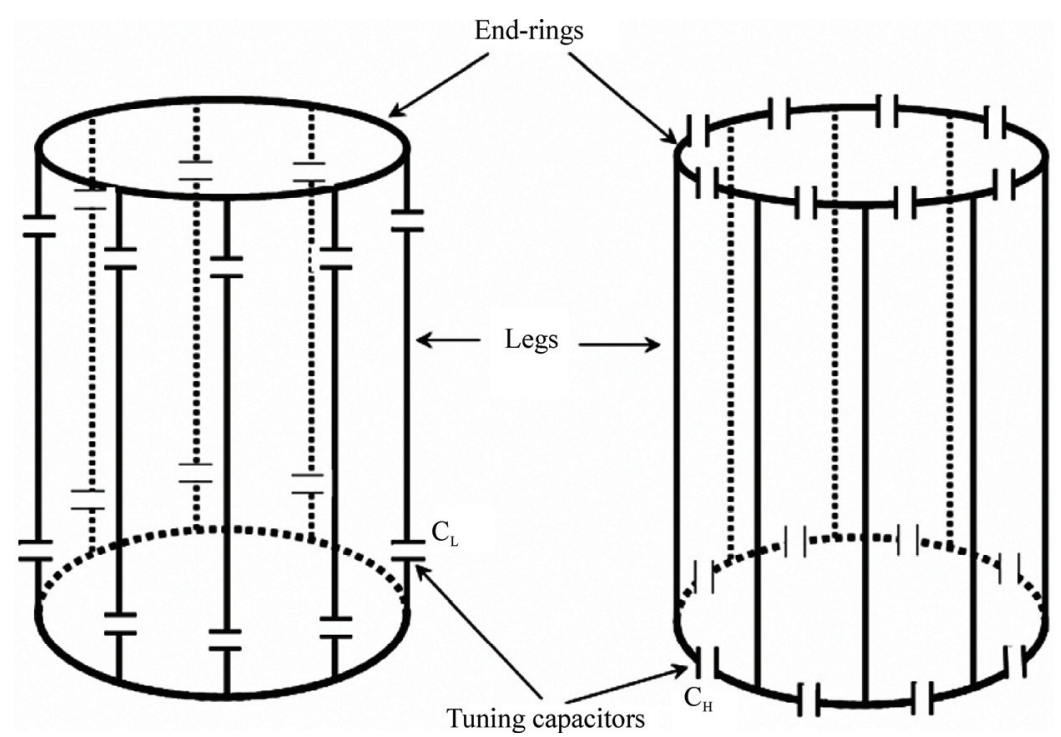

Figure 1. Spatial representation of the low-pass (left) and high-pass (right) birdcage resonators. $\mathrm{C}_{\mathrm{H}}$ (high-pass) and $\mathrm{C}_{\mathrm{L}}$ (low-pass) are the tuning capacitors.

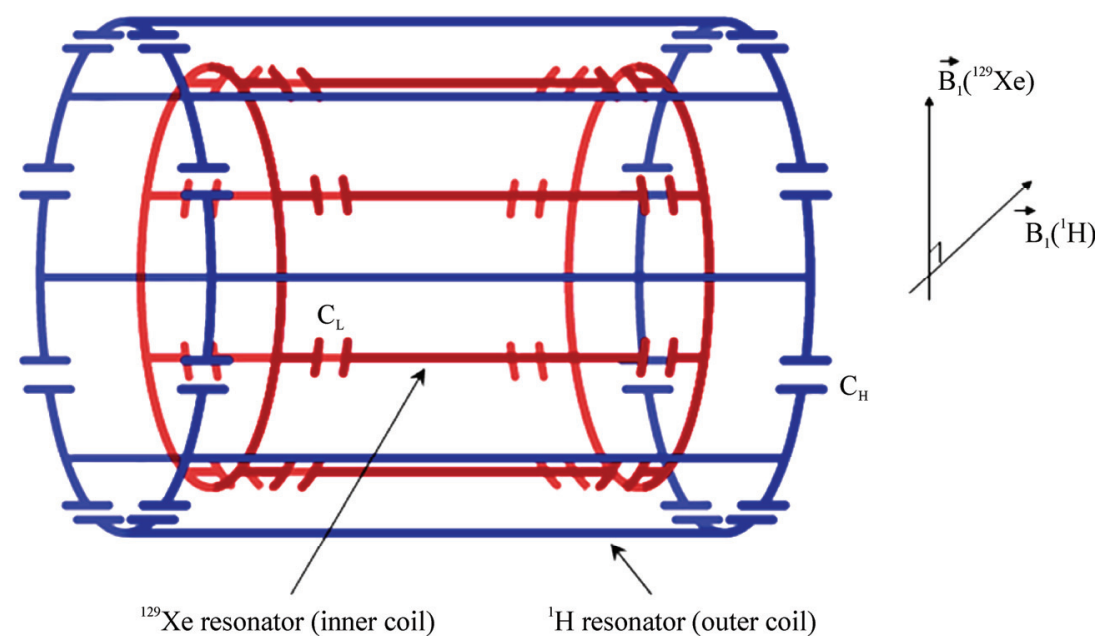

Figure 2. A simplified scheme of the developed imbricate birdcage resonators. The inner coil is low-pass and the outer one is high-pass. In linear polarization operation, the coils should be derived in such a way that their $\mathrm{B}_{1}$ fields are orthogonal allowing efficient geometric decoupling between the resonators. 
cage was made longer than the inner one, minimizing coupling between the pairs of end rings. Realistically, one cannot achieve perfect isolation between the two coils since the legs of the coils are in close proximity. The coupling is only reduced. For our application, since the resonance frequencies of the coils are far away from each others, this geometric decoupling could be accepted. Quantitative measurements of the isolation between the two coils are given in Section 3.

The calculation of the necessary tuning capacitances for both coils was performed using the free BirdcageBuilder software from the Penn State University [18]. The practical used values for these tuning capacitors were close to the calculated ones.

Variables capacitors were used to perform a final fine tuning when the coils are placed inside the magnet and loaded by the sample (Figure 3). For the low-pass birdcage, the fine tuning is achieved by adjusting tow diametrically opposed capacitors $\left(\mathrm{C}_{\mathrm{LT}}\right.$ in Figure 3$)$. This method allows only a limited tuning range. Otherwise, it destroys the original ideal sinusoidal current distribution between the legs [17]. After testing the resonator inside the magnet and with different loads (phantoms, rats), we limited the tunable range of the resonator to sufficient range of about $500 \mathrm{kHz}$, so as the current deviation from its ideal is less than $10 \%$ [17].

For the high-pass resonator, the fine tuning is accomplished by connecting two diametrically opposed points of one end-ring by a tuning variable capacitor $\mathrm{C}_{\mathrm{HT}}$. In practice, the connection of these points is made by adding a second circular loop, parallel to the end-ring as shown in Figure 3. To preserve the symmetry of the birdcage, the adjustable tuning capacitors $\mathrm{C}_{\mathrm{HT}}$ was balanced by a fixed capacitor of $8.2 \mathrm{pF}$ connected across the second end ring using a circular loop (not shown in Figure 3).

Both coils were derived in linear polarization operation in such a way that theirs $B_{1}$ fields are orthogonal as it was shown on Figure 2. We used balanced capacitive coupling to match their impedances to the real value of 50 at the working frequency [17]. The coupling scheme of the low-pass resonator is shown together with the active decoupling circuitry in Figure 4. In this scheme, the capacitive coupling, accomplished through the capacitors $\mathrm{C}_{\mathrm{T}}$ and $\mathrm{C}_{\mathrm{M} 1}, \mathrm{C}_{\mathrm{M} 2}$ and $\mathrm{C}_{\mathrm{M} 3}$, is preceded by a balun (balanced-unbalanced). This balun is required for the probe circuit, not only due to the necessity of equilibrating the probe coil and consequently minimizing dielectric coil losses in the sample, but also for blocking unwanted common mode currents. The balun we built is widely used in radio technologies $[17,19]$. It consists of a simple "trap" circuit where the loop formed by the coaxial cable is tuned by a capacitor, $\mathrm{C}_{\mathrm{B}}$, to form a high impedance circuit at the xenon frequency.

The capacitive coupling scheme for the low-pass birdcage is straightforward to be generalized to proton resonator. It will not be given here, but the interesting reader may simply write to the author for more details.

As it was outlined in a previous paragraph, the transmit-only birdcage coil for ${ }^{129} \mathrm{Xe}$ (inner coil) must be decoupled from the receive-only surface coil (described in the next section). During transmission, this is mandatory since a coupling between the coils would result in deleterious consequences for the homogeneity of the $\mathrm{B}_{1}$ field and in a possible destruction of the receive coil. During receiving, part of the electromagnetic energy received by the receive coil will be dissipated in the transmit volume

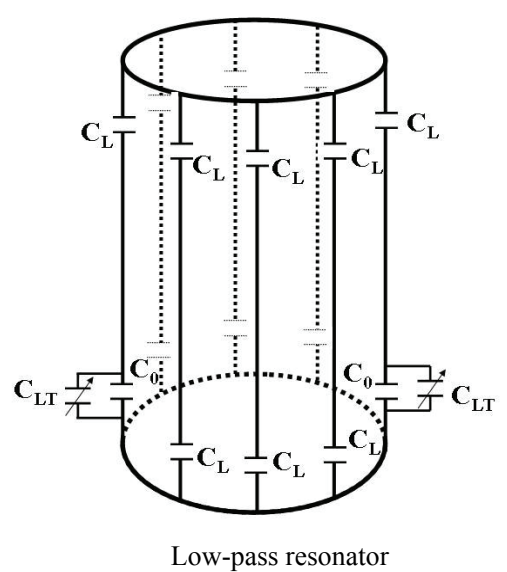

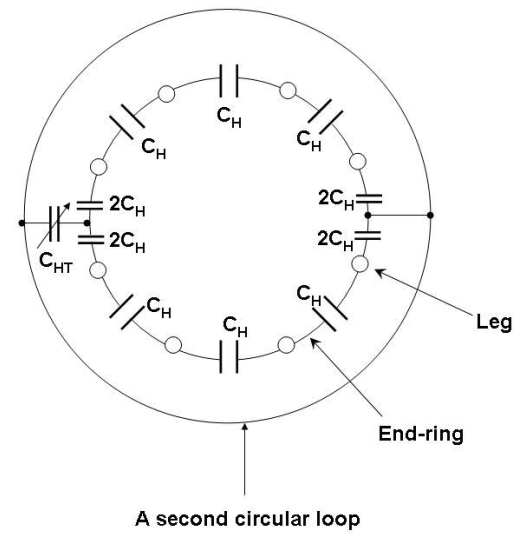

High-pass resonator

Figure 3. The fine tuning of the resonators. Low-pass resonator: $\mathrm{C}_{\mathrm{L}}=340 \mathrm{pF}(220 \mathrm{pF}+120 \mathrm{pF}$ chip capacitors from American Technical Ceramics Corp. ATC), $\mathrm{C}_{\mathrm{LT}}=1.5-55 \mathrm{pF}$ (adjustable capacitor NMAT55HVE from Voltronics). High-pass resonator (only one end-ring is shown): $\mathrm{C}_{\mathrm{H}}=40 \mathrm{pF}$ (18 $\mathrm{pF}+12 \mathrm{pF}$ chip capacitors from ATC Corp.), $\mathrm{C}_{\mathrm{HT}}=1-15 \mathrm{pF}$ (adjustable capacitor NMAT15HVE from Voltronics). To preserve the symmetry of the high-pass birdcage, a tuning capacitor (which balances $\mathrm{C}_{\mathrm{HT}}$ ) should be connected across the second end-ring (not shown in the figure). We used a fixed chip capacitor of $8.2 \mathrm{pF}$ to balance $\mathrm{C}_{\mathrm{HT}}$. 


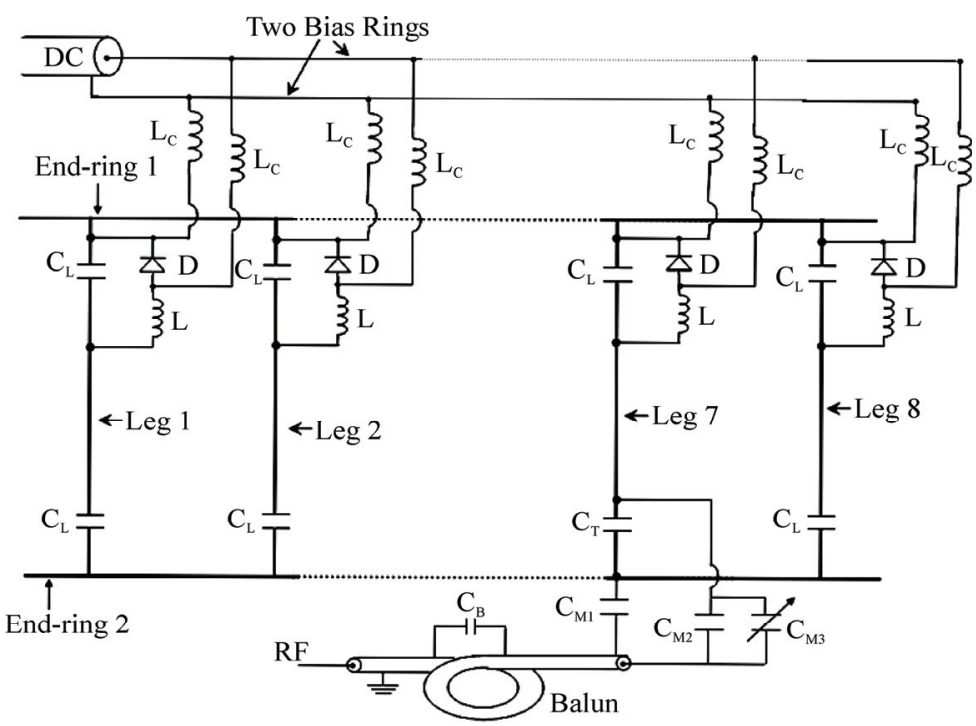

Figure 4. The ${ }^{129} \mathrm{Xe}$ birdcage electrical circuit associated with the active decoupling circuitry. $\mathrm{C}_{\mathrm{L}}(220+120 \mathrm{pF}$ from ATC Corp. $)$ are tuning capacitors, $\mathrm{L}_{\mathrm{C}}$ $(8 \mu \mathrm{H}+4 \mathrm{x} 1 \mu \mathrm{H}$ from CoilCraft) are the RF choke inductors, $L(90 \mathrm{nH})$ are parallel inductors (from CoilCraft) and D are PIN diodes (UM4006 from Microsemi Corp). Matching is achieved by the use of balanced capacitive coupling through ATC chip capacitors: $\mathrm{C}_{\mathrm{T}}=220 \mathrm{pF}, \mathrm{C}_{\mathrm{M} 1}=120 \mathrm{pF}, \mathrm{C}_{\mathrm{M} 2}=53 \mathrm{pF}(10$ $\mathrm{pF}+10 \mathrm{pF}+33 \mathrm{pF})$. For fine matching, $\mathrm{C}_{\mathrm{M} 3}$ is a variable capacitor with capacitance between $1.5 \mathrm{pF}$ and $55 \mathrm{pF}$ (NMAT55HVE from Voltronics). Notice that $C_{T}+C_{M 2}+C_{M 3} \approx C_{L}$, which is with good agreement with balanced capacitive coupling condition [17]. The adjustable $1.5 \mathrm{pF}-55 \mathrm{pF}$ tuning capacitors $\mathrm{C}_{\mathrm{LT}}$ for fine tuning are not shown (see Figure 3). The tuning capacitor $\mathrm{C}_{\mathrm{B}}$ for the balun $\mathrm{C}_{\mathrm{B}}=180 \mathrm{pF}+120 \mathrm{pF}$.

coil resulting in SNR and sensitivity losses. Therefore, the interaction of the volume resonator and the receive coil must be cancelled out.

Decoupling can be achieved in principle geometrically (as in the case of the two concentric birdcages). However, when the coils are tuned to the same frequency, it is difficult in practice to perfectly decouple them by simply adjusting their mutual orientation. Hence, geometric decoupling should be accompanied or replaced by another effective mean that cancels out or, at least, minimize the induced currents in each coil.

This can actually be done by shifting the resonance frequency of one coil with respect to the other during appropriate periods. This detuning method is well described and used to detune a birdcage coil in [20] for example. In this relatively simple method, usually used in commercial coils, the current in the detuned coil is not cancelled out but only reduced.

In more efficient design, the current in the resonator is cancelled out at the working frequency, improving the isolation to a high level. In such a design, which is based on the "pole insertion" technique, an inductance is connected, using switching diodes, in parallel to one or more (depending on the coil structure and type) tuning capacitors, constituting a parallel resonant circuit (pole insertion). If the pole is well-tuned to the working frequency, the resulting "trap" circuit becomes an efficient blocking RF current device. This method was described for the case of a single-tuned high-pass birdcage [21]. In our prototype, we demonstrate that this method can be generalized to the case of a low-pass birdcage which has not been published.

We realized an active decoupling through the use of high voltage PIN diodes (UM4006, Microsemi Corp) distributed across one set of the legs capacitors. The schematic of the decoupling circuitry is shown in Figure 4.

For each leg, two bias rails apply the appropriate DC bias, through the RF choke inductors $L_{C}$ to the PIN diode which, when it is forward-biased, switches an inductor $L$ in parallel with the tuning capacitor $C_{L}$. If the value of the inductor $L$ is correctly chosen $\left(L \cdot C_{L} \cdot \omega_{0}^{2}=1\right)$, the resulting parallel circuit presents a high impedance at the working frequency so as the RF current is cancelled out in each leg and the transmit volume coil is "off" (receiving period). When the diode is reverse-biased, it forms an open circuit and the inductor $L$ is disconnected. In this case, the coil is "on" (transmission period).

The inductors $L_{C}$ in the direct DC path serve as chokes and ensure "isolation" between the RF part and 


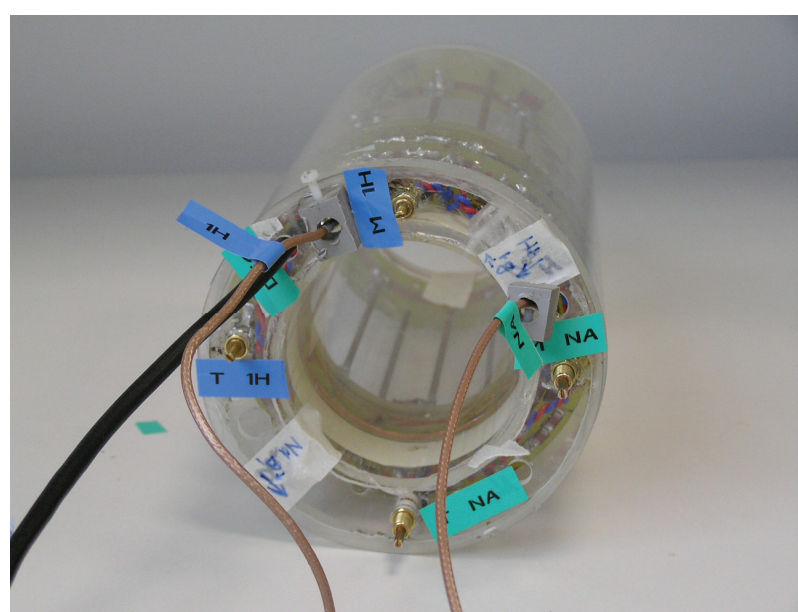

Figure 5. The double-tuned volume coil. We can see (right picture) the four variable tuning and matching capacitors for the two channels.

the DC bias circuit by minimizing RF currents on the DC path.

Figure 5 shows the double-tuned volume coil.

\subsection{The Receive-Only Single-Tuned Coil}

A surface coil was chosen for the receive-only function. It consists of a single circular loop of $4 \mathrm{~cm}$ of diameter (Figure 6). The tuning capacitor $C_{t}$ was distributed into two series capacitors $\left(2 C_{t}\right)$ positioned in two different physical sites of the loop to reduce the losses associated with the electric field generated by the coil $[22,23]$.

The decoupling of this coil from the transmit volume coil was achieved using the "pole insertion" method as illustrated in Figure 6. The PIN diode switches or not in parallel the inductor $L_{s}$ across one of the distributed tune capacitors $\left(2 C_{t}\right)$. During the transmission period the coil is "off" (diode is forward-biased), while it is "on" during the receiving period (diode is reverse biased).

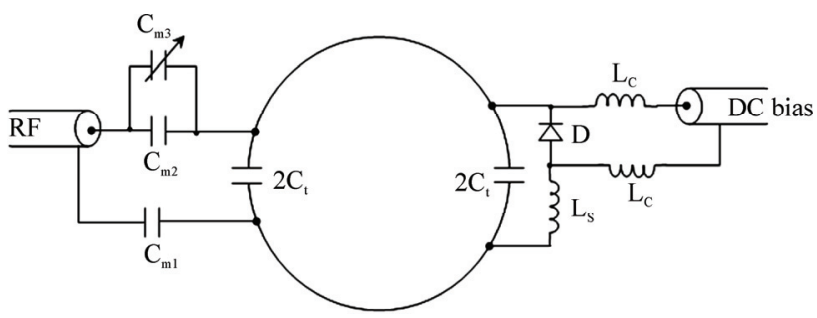

Figure 6. The receive-only coil and its active decoupling circuitry. The fixed tuning is $2 C_{t}=2 \times 220 \mathrm{pF}+2 \times 150 \mathrm{pF}+100 \mathrm{pF}$ (chip capacitors from ATC). The adjustable capacitor of 1.5-55 $\mathrm{pF}$ (NMAT55HVE from Voltronics) is not shown. The matching capacitors are: $C_{m l}=68 \mathrm{pF}+10 \mathrm{pF}, C_{m 2}=56 \mathrm{pF} C_{m 3}=1.5-55$ $\mathrm{pF}$ (adjustable capacitors NMAT55HVE from Voltronics). $L s=40 \mathrm{nH}$ (3 parallel $120 \mathrm{nH}$ inductor from CoilCraft) is the parallel inductor. D is a PIN diode (UM4006 from Microsemi Corp). $L c=8 \mu \mathrm{H}+5 \mathrm{x} 1 \mu \mathrm{H}$ are RF choke inductors (from CoilCraft).

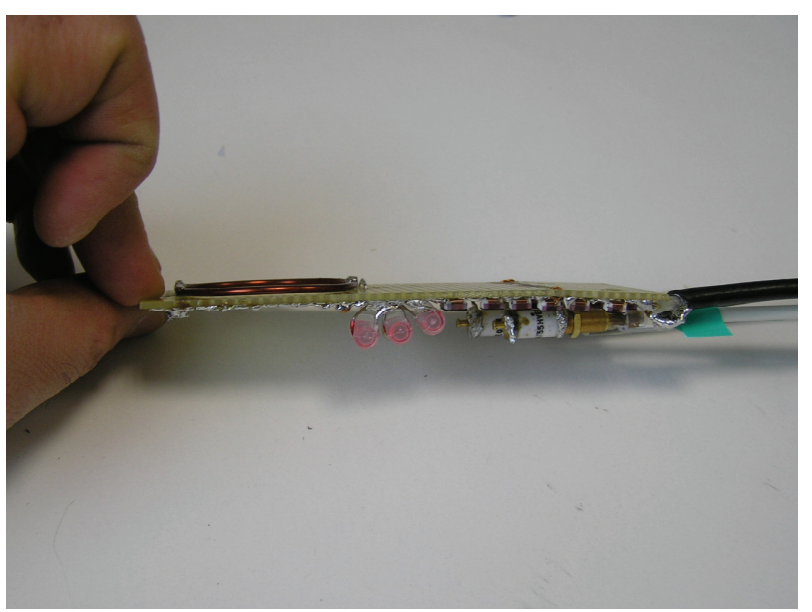

Figure 7. The receive-only surface coil and its circuitry. The loop was fixed on one side of a printed board and all components were soldered on the other side.

Notice finally that the receive-only coil was matched using balanced capacitive coupling (capacitors $C_{m 1}, C_{m 2}$, and $C_{m 3}$ in Figure 6). RF choke inductors $L_{C}$ are used to "separate" the RF circuit from DC bias one. Figure 7 shows the developed single-tuned receive only coil.

\subsection{The $T / R$ Driver}

PIN diodes for both transmit and receive-only coils were biased through the use of a home-made T/R driver. This driver provides the gated DC bias necessary for electronically turning coils "on" and "off". We designed the driver with a gated input (a TTL compatible signal supplied by the NMR spectrometer) and two separate TX and RX bias sections. The TX stage applies a $-12 \mathrm{Vdc}$ bias (PIN diodes are reverse biased and transmit volume coil is "on") and a 5 Vdc bias (PIN diodes are forward biased and transmit coil is "off"). The RX stage applies $-12 \mathrm{Vdc}$ bias (PIN diode opened and receive-only coil is "on") and a 5 Vdc bias (PIN diode closed and the coil is "off"). The driver contains current limitation resistors to limit the maximum output current to $1 \mathrm{~A}$ for both TX and RX sections.

The flexibility of the driver allows the possibility to turn "off" and "on" both coils by using a manual switch (without the need to a TTL signal) for fine tuning and matching purposes inside the magnet. Another manual switch allows also the use of volume ${ }^{129} \mathrm{Xe}$ coil for both transmission and receiving. In this situation, the receiveonly coil is switched "off" during all the experiment (PIN diode forward biased).The design of the driver was based on the use of bipolar transistors.

\section{RESULTS AND DISCUSSIONS}

Although this specific probe-head was initially developed for the MRI of $\mathrm{HP}^{129} \mathrm{Xe}$, we performed all the tests 
and all the validation experiments at proton and sodium-23 $\left({ }^{23} \mathrm{Na}\right)$ frequencies. Actually, it is important to notice that the magnetization of $\mathrm{HP}^{129} \mathrm{Xe}$ is artificial and it is therefore not renewable when destroyed by the RF excitation pulses. Clearly, this is not a convenient situation for coil characterization and adjustments. Sodium frequency at 2.35 Tesla $(26.4 \mathrm{MHz})$ is very close to the ${ }^{129} \mathrm{Xe}$ one $(27.7 \mathrm{MHz})$. We could therefore consider that all the tests and measurements conducted at the sodium frequency would be valid at the ${ }^{129} \mathrm{Xe}$ frequency. Validation at the HP ${ }^{129} \mathrm{Xe}$ frequency for in vivo applications will make the subject of a future work.

During all the tests, the receive-only surface coil was placed inside the volume probe in such a way that geometric decoupling between it and the proton volume coil, is always achieved. It is clear here that no geometric decoupling could be realized between the receive-only coil and the sodium volume coil. Only active decoupling between these coils is achieved.

As it was mentioned in a previous section, a singletuned birdcage will resonate in several modes, with the most useful to MRI being Mode 1 . In this mode, a very homogenous transverse magnetic field is created by the sinusoidal distribution of the currents in the legs. It is important for our application to know whether or not this distribution of currents was disturbed by the doubletuned configuration, and consequently to know whether or not the $\mathrm{B}_{1}$ homogeneity, especially for the ${ }^{23} \mathrm{Na}$ coil, is preserved. Moreover, we are interested in knowing if the receive-only coil would or not disturb the sinusoidal distribution of the currents of the proton coil since only geometric decoupling was realized between these coils. Also, we wanted to know if the only-active decoupling between this receive-only coil and sodium volume coil would be sufficient to preserve the homogeneity of the $\mathrm{B}_{1}$ without the need of geometric decoupling. All these information could be obtained by measuring the currents in the legs of both birdcages at the proton and sodium frequencies. We measured the currents distribution in each coil by placing a small sense loop adjacent to the legs. The receive-only coil was switched off during all measurements. At the sodium frequency, we measured the currents in the legs of sodium birdcage (inner resonator), as well as the currents induced in the proton legs (outer resonator). Similarly, these currents were measured at the proton frequency. Measurements are shown in Figure 8.

At the sodium frequency, the currents in the sodium birdcage (inner coil) follow a cosine distribution as one moves radially around the coil. Small currents are induced in the proton resonator (outer coil). These currents are in phase with and add to the currents in the sodium coil. From this information, one can expect that the transverse magnetic field $\mathrm{B}_{1}$ created by the sodium coil would still homogenous and would not be disturbed by the proton coil. At the proton frequency, the measurements show strong out of phase currents in the sodium coil. Despite the presence of these currents, the currents in the proton legs still follow the desired sinusoidal distribution as one moves radially around the coil. The coil still creates a homogenous $\mathrm{B}_{1}$ field. The presence of the out of phase currents in the sodium coil will result, off course, in losses in the efficiency of the proton coil when compared to a single-tuned one.

With the simple measurement method of currents which was based on the use a small sense loop, no changes on currents distribution were observed with or without the presence of the surface coil. One could then argue that the presence of the receive-only coil, in its "off" state, does not disturb the homogeneity maps of $\mathrm{B}_{1}$ fields of both sodium and proton birdcages.

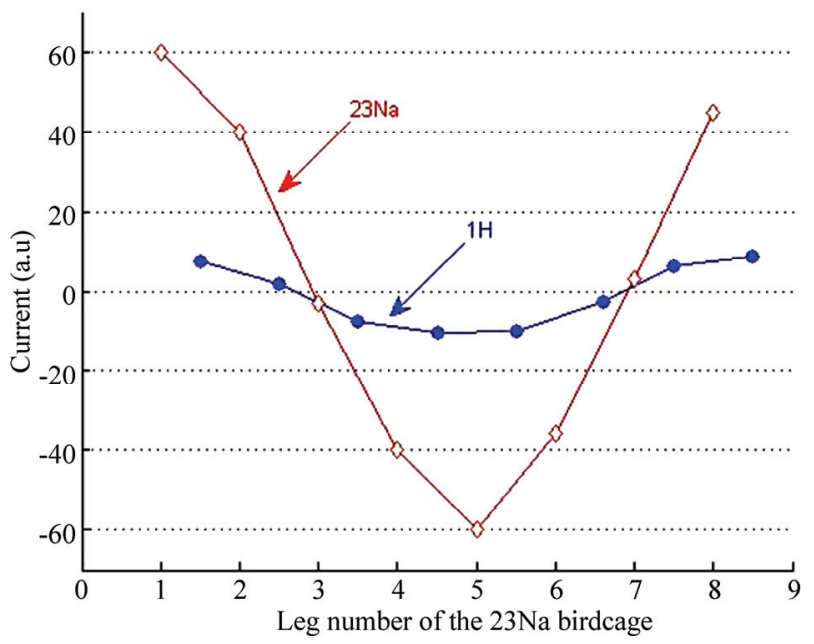

(a) Currents distribution at sodium frequency

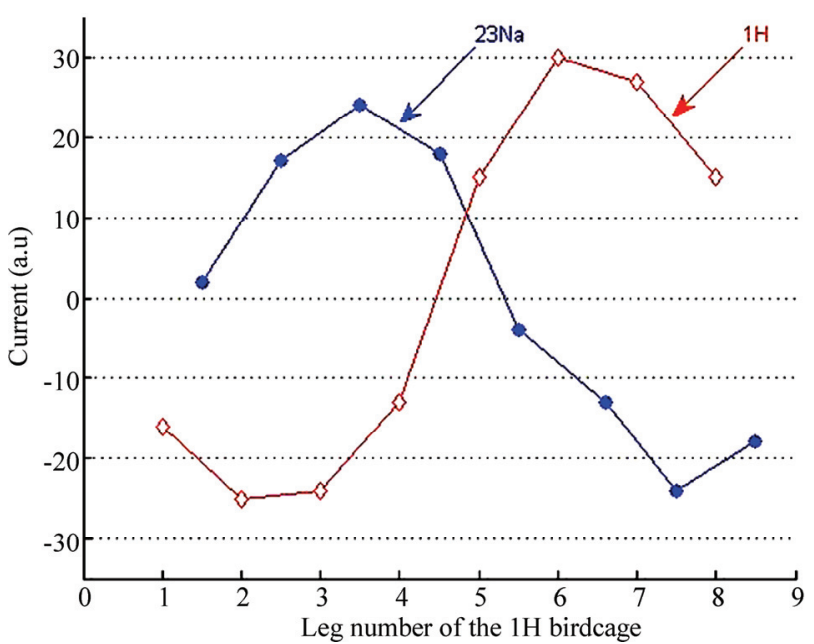

(b) Currents distribution at proton frequency

Figure 8. Currents distribution in the resonators at (a) sodium and (b) proton frequencies. 
It's important to evaluate the degree of isolation between the coils. We have conducted isolation measurements between the different coils using a network analyzer. At sodium frequency, the isolation between the proton and the sodium birdcages was greater than $25 \mathrm{~dB}$. At proton frequency, the isolation was lowered to about $16 \mathrm{~dB}$. These measurements are in agreement with current measurements. Actually, at the sodium frequency, very small currents are induced in the proton coil and one can expect good isolation. Conversely, at the proton frequency, where large currents are induced in the sodium coil, the isolation is naturally lowered.

Isolation measurements between the receive-only coil and the two volume coils were also conducted. At the proton frequency, the isolation between the proton birdcage and the surface coil was about $26 \mathrm{~dB}$. This isolation is very good and confirms that the cosine current distribution is not, or little, disturbed by the surface coil. Geometric decoupling between these two coils should sufficient for our application. Isolation between the sodium birdcage and the receive-only coil was also measured, with the only-active decoupling, to be about $31 \mathrm{~dB}$. This excellent isolation between the two coils is essential in maintaining a transmit field homogeneity and in retaining the high sensitivity of the surface coil.

We have realized MRI images using the developed probe. In the first time, images were acquired using phantoms. A variety of phantoms was used to test the developed probe in various conditions and configurations.

The first series of images was realized using the proton birdcage and the sodium birdcage biased as transmit/receive coils. The surface coil was in its "off" state during both transmitting and receiving periods. The phantom (see Figure 9) was consisting of three cylindrical tubes with a same height $(50 \mathrm{~mm})$. The biggest tube $(27$ $\mathrm{mm}$ of inner diameter) was filled with saline solution (pure water saturated with $\mathrm{NaCl}$ ). The two small tubes (12.5 $\mathrm{mm}$ of inner diameter) were filled with pure water.

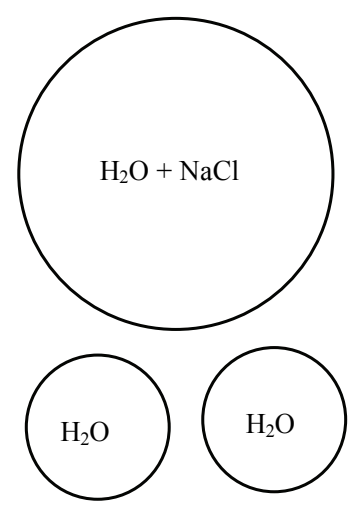

Figure 9. The cross-section (drawn to scale) of the phantom used to validate the double-tune birdcages.

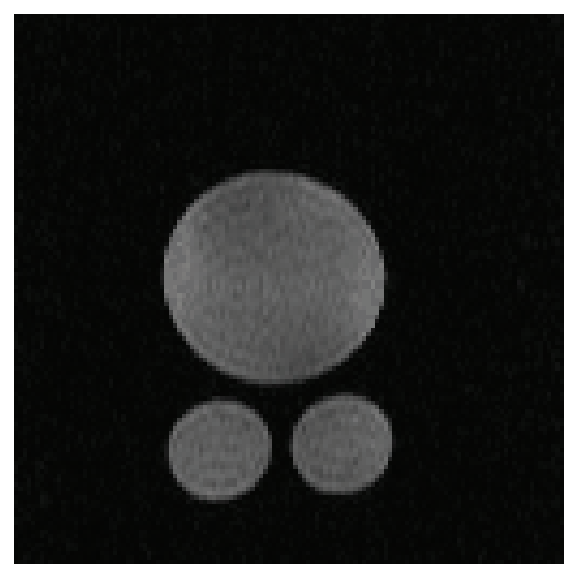

(a)

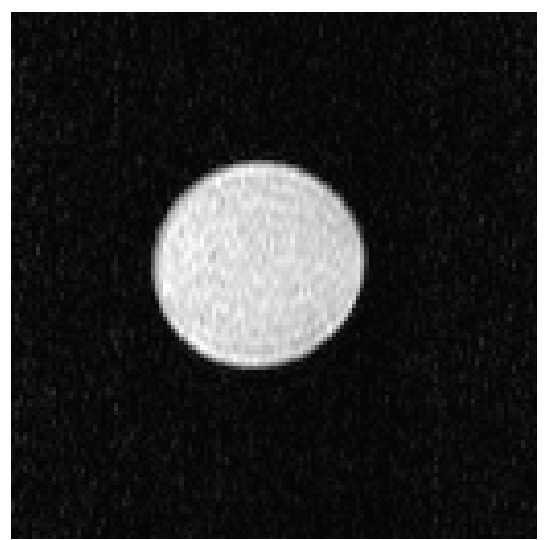

(b)

Figure 10. The cross-sectional (axial) MRI images (displayed to scale) of a phantom acquired at (a) proton frequency (100 $\mathrm{MHz}$ ) and (b) sodium frequency (26.4 MHz). A spin echo pulse sequence was used to acquire images. FOV (Field of View) $=70 \times 70 \mathrm{~mm}^{2}$, repetition time $\mathrm{TR}=1000 \mathrm{~ms}$, echo time $\mathrm{TE}=31 \mathrm{~ms}$, flip angle $=90^{\circ}$. Slice thickness for proton images $=2$ $\mathrm{mm}$. Sodium images were acquired with 2 signal averages and without slice selection.

Figure 10 shows the cross-sectional MRI spin-echo images of the phantom obtained at $100 \mathrm{MHz}$ and 26.4 MHz with a field of view (FOV) of $70 \times 70 \mathrm{~mm}^{2}$ and an acquisition matrix of $128 \times 64$. Before transformation of the acquired data to the image space, zero filling to $128 \times 128$ was applied.

In these images, we can see the high homogeneity of the $\mathrm{B}_{1}$ produced at $26.4 \mathrm{MHz}$. This confirms the currents measurements addressed above. The sodium birdcage was not disturbed by the proton coil. The homogeneity of the $\mathrm{B}_{1}$ created at $100 \mathrm{MHz}$ is shown to be also sufficient.

The second series of images was realized with the proton birdcage used for both transmitting and receiving. The sodium birdcage was biased as a transmit-only coil, while the surface sodium coil was biased as a receiveonly coil. This is actually the final configuration of use of the developed probe for our application. A three- 
compartment cylindrical phantom was used for this experiment (Figure 11). It was formed of a small tube (inner diameter $=10.5 \mathrm{~mm}$ ) filled with pure water and placed approximately diagonally inside a larger tube (inner diameter $=28 \mathrm{~mm}$ ) filled with a saline solution. Between the two tubes, we have intentionally conserved an empty space with a variable width as illustrated by Figure 11.

Cross-sectional MRI spin-echo images of this threecompartment phantom were acquired at $100 \mathrm{MHz}$ and at $26.4 \mathrm{MHz}$ (see Figure12) with a FOV of $50 \times 50 \mathrm{~mm}^{2}$ and an acquisition matrix of $128 \times 64$. Before transformation of the acquired data to the image space, zero filling to $128 \times 128$ was applied.

We can recognize on the sodium images the typical sensitivity of a surface coil. This sensitivity decreases when one moves away from the center of the coil. We can, off course, expect a better signal-to-noise ratio with a surface coil when compared with a volume coil. This is actually well-known and no more signal-to-noise ratio characterization is necessary.

The validation of the developed probe was further demonstrated in vivo in the rat brain. Firstly, spin-echo images were obtained at the proton frequency. Figure 13 (respectively Figure 14) shows a set of cross-sectional (respectively coronal) images.

Notice that, in the configuration of the developed probe, the filling factor of the outer birdcage (proton coil) is not optimum for the outer coil. Consequently, its SNR performances are relatively reduced when compared with those that could be obtained by a single simple-tuned birdcage. Actually, it is well-known that the SNR is directly proportional to square root of the coil filling factor. This point does not handicap the probe since high SNR performances of the ${ }^{1} \mathrm{H}$ coil are not an issue in our ap- plication. Actually, the ${ }^{1} \mathrm{H}$ will be only used for acquiring localization (anatomic) images where signal averaging could be applied to enhance the SNR. For shimming purposes, there is no need to have a high SNR.

The developed probe was also tested in vivo at the sodium frequency on the same rat. The sodium birdcage was biased as a transmit-only coil, while the sodium surface coil was biased as a receive-only coil. Our goal here is not to have sodium images of the rat brain, but only to demonstrate a basic functioning of the sodium coil in vivo. Only sodium NMR signal and spectra in the rat brain were acquired as it is shown in Figure 15. We obtained the typical sodium spectra of sodium in the rat brain with a typical peak width of few ppm [24].

In vivo sodium MRI images were difficult to obtain with classical MRI pulse sequences (spin-echo, gradient-echo) except if an incredible number of signal averages (more than 20 hours of acquisition time) is used. Actually, because of the short $\mathrm{T} 2$ transverse relaxation time components of sodium in the rat brain $(0.7-3 \mathrm{~ms}$ or $16-30 \mathrm{~ms}$ depending on the value of the magnetic field), the acquisition of sodium images requires the imple-

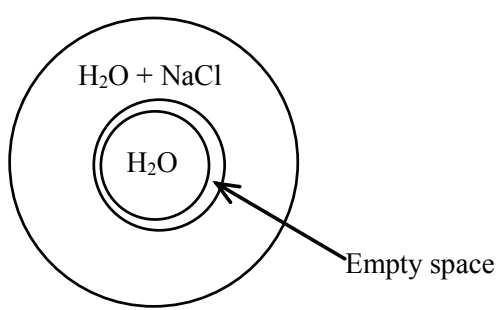

Figure 11. A schematic diagram (drawn to scale) of the cross-section of the three-compartment phantom.

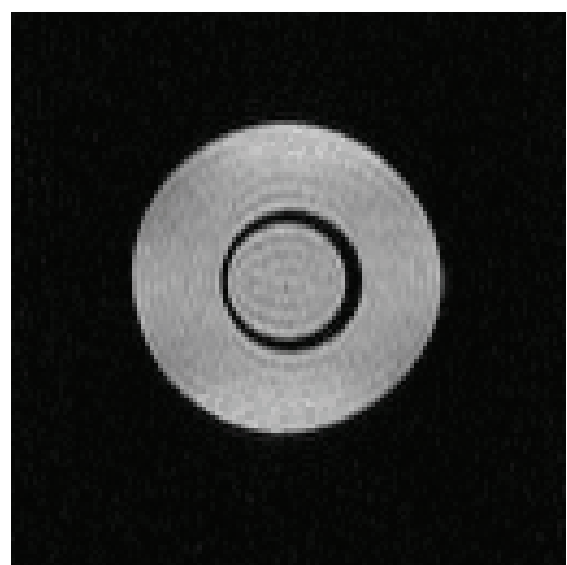

(a)

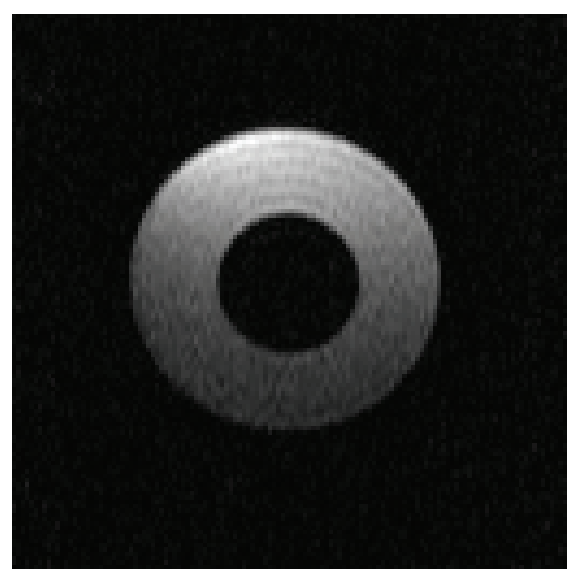

(b)

Figure 12. Cross-sectional (axial) MRI images (displayed to scale) of the three-compartment phantom acquired at (a) proton frequency $(100 \mathrm{MHz})$ and (b) sodium frequency $(26.4 \mathrm{MHz})$. A spin echo pulse sequence was used to acquire images. $\mathrm{FOV}=50 \times 50 \mathrm{~mm}^{2}$, flip angle $=90^{\circ}$, echo time $\mathrm{TE}=30 \mathrm{~ms}$, repetition time $\mathrm{TR}=2000 \mathrm{~ms}$ for proton and $300 \mathrm{~ms}$ for sodium. Slice thickness for proton images $=2 \mathrm{~mm}$. Sodium images were acquired with no slice selection and with no signal averaging. 

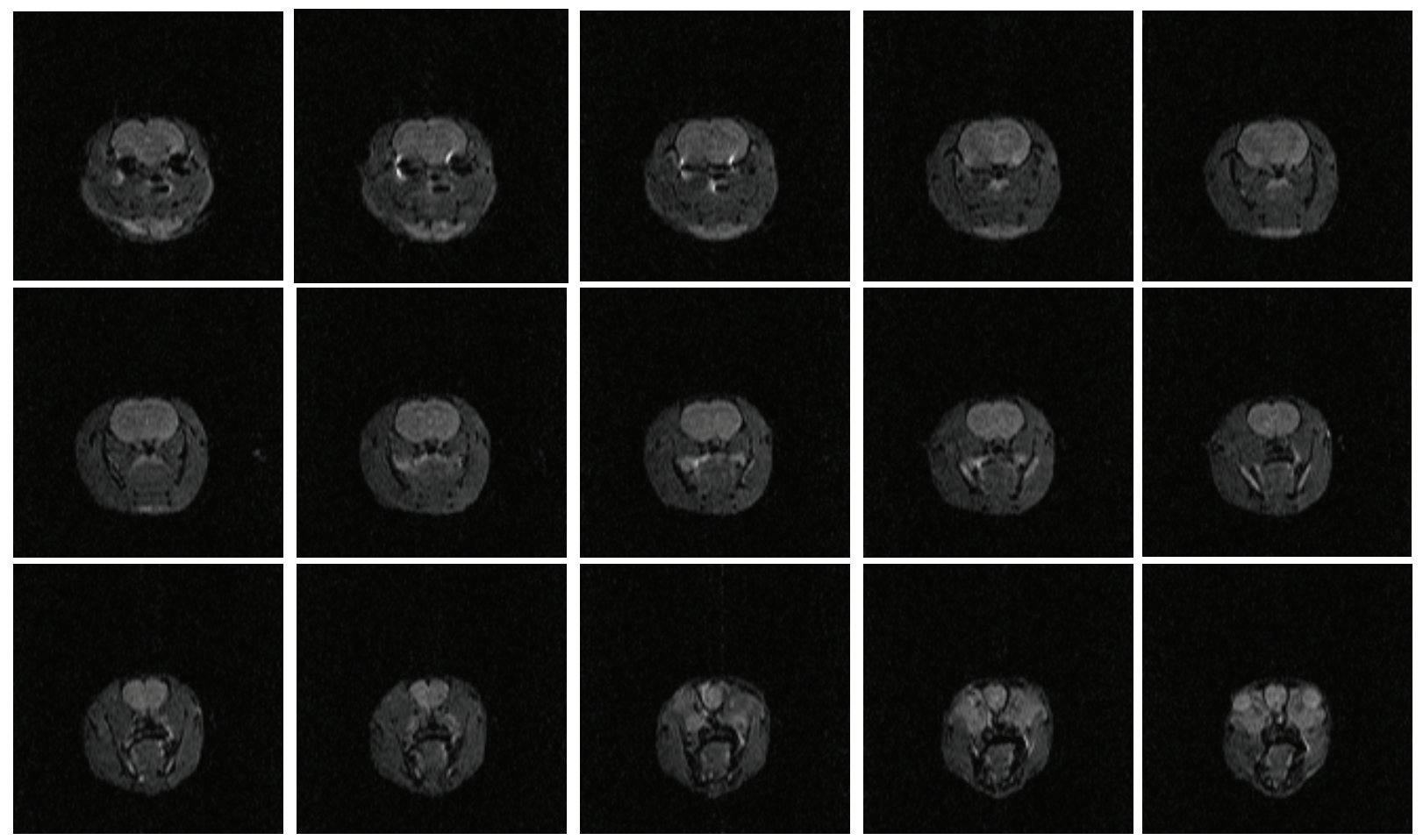

Figure 13. Cross-sectional MRI images of the rat brain acquired at $100 \mathrm{MHz}$. A spin echo pulse sequence was used to acquire images. FOV (Field of View) $=60 \times 60 \mathrm{~mm}^{2}$, flip angle $=90^{\circ}$, echo time $\mathrm{TE}=30 \mathrm{~ms}$, repetition time $\mathrm{TR}=4000 \mathrm{~ms}$, slice thickness $=1 \mathrm{~mm}$. Acquisition matrix was $128 \times 64$. Before transformation of the acquired data to the image space, zero filling to $128 \times 128$ was applied.
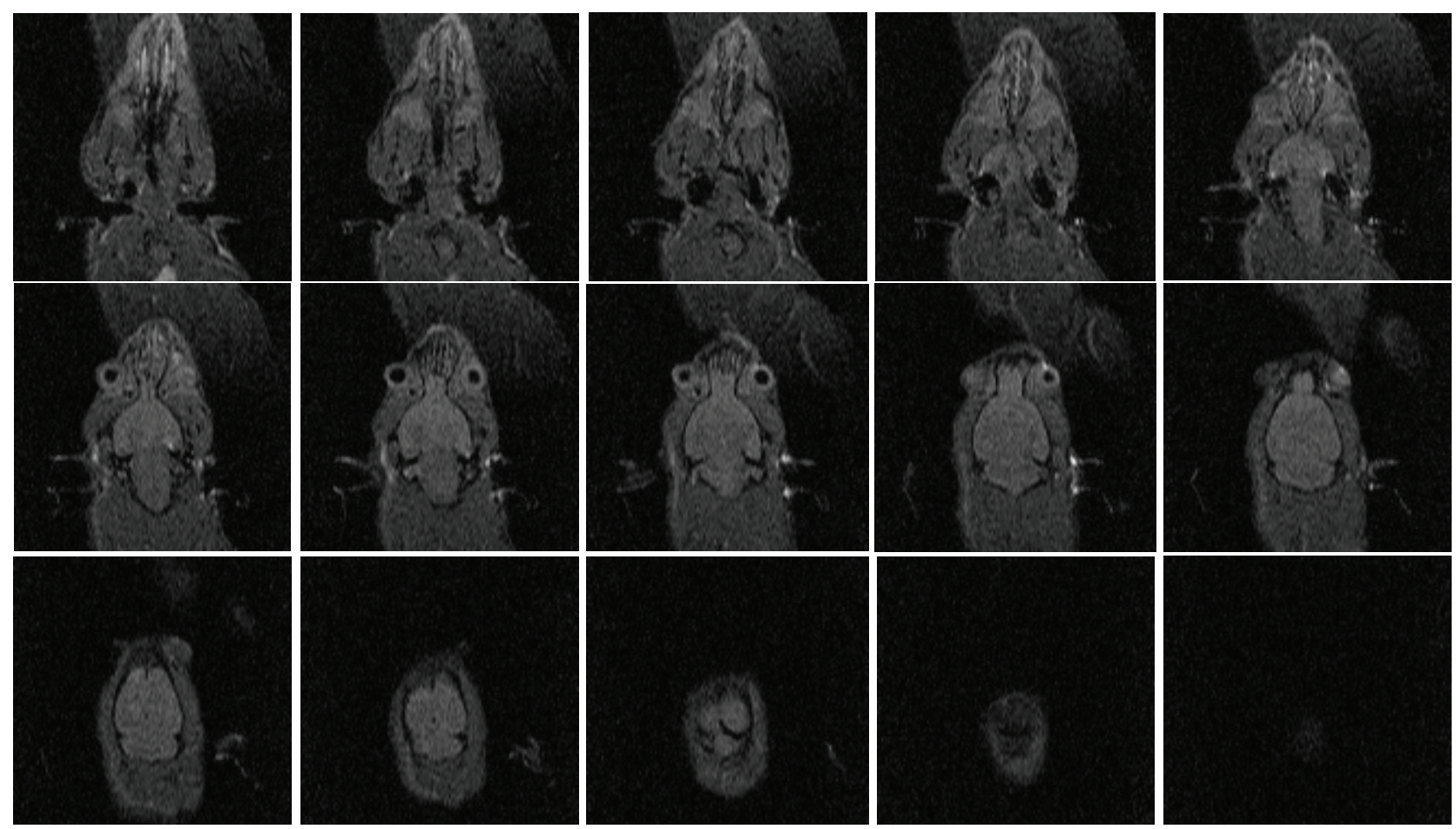

Figure 14. Coronal MRI images of the rat brain acquired at $100 \mathrm{MHz}$. A spin echo pulse sequence was used to acquire images. FOV (Field of View) $=60 \times 60 \mathrm{~mm}^{2}$, flip angle $=90^{\circ}$, echo time $\mathrm{TE}=17 \mathrm{~ms}$, repetition time $\mathrm{TR}=4000 \mathrm{~ms}$, slice thickness $=1 \mathrm{~mm}$. Acquisition matrix was $128 \times 64$. Before transformation of the acquired data to the image space, zero filling to $128 \times 128$ was applied. 


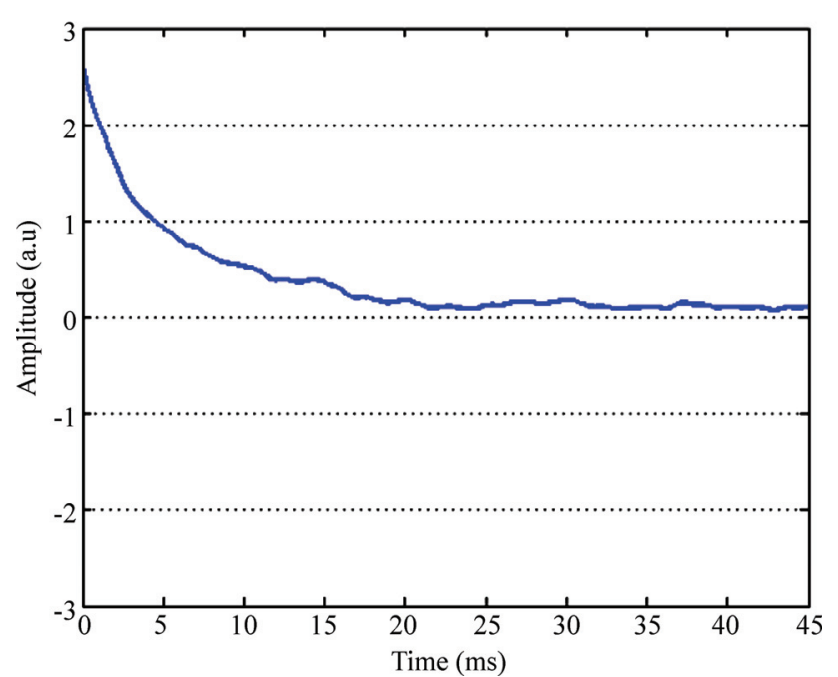

(a)

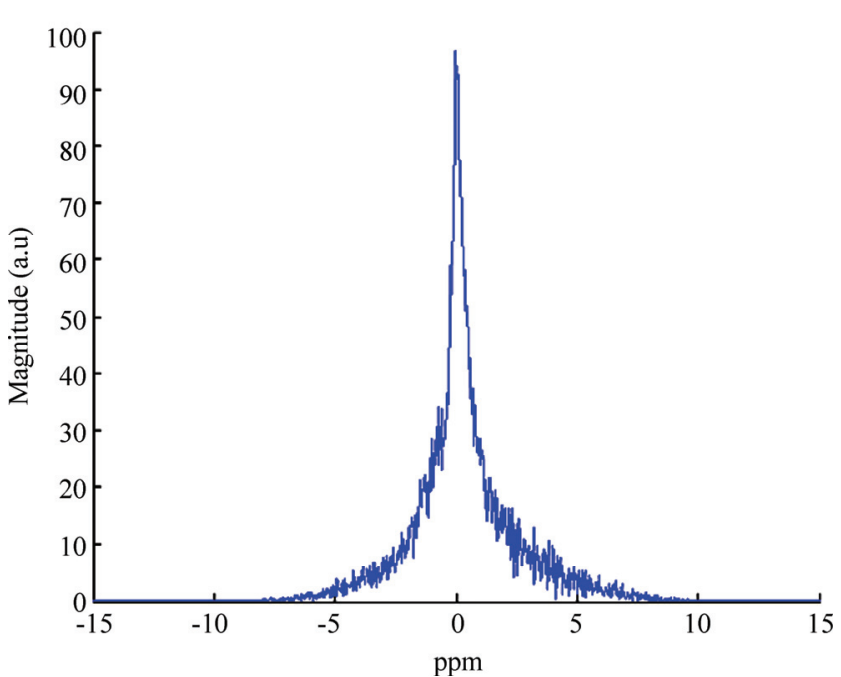

(b)

Figure 15. In-vivo sodium (a) NMR signal and (b) its spectrum in the rat brain. A hard non-selective excitation pulse and 512 signal averages were used.

mentation of dedicated three-dimensional (3D) and short echo-time pulse sequences (3D back-projection sequence and 3D GRE for example) [14,25]. The implementation of $3 \mathrm{D}$ sequences is out of the scope of our research topics.

Nevertheless, we think that our probe presents no intrinsic limitation for acquiring in-vivo sodium images. The only limitation is the available MRI sequence. To demonstrate this without the need to implement new sequences, we tried to acquire sodium image of a phantom containing a sodium concentration as low as those which are generally present in vivo. The sodium transverse relaxation time of the phantom must be longer than its in-vivo value to allow the use of a classical spin-echo sequence. So, we used a syringe filled with $20 \mathrm{ml}$ of a physiological serum. The sodium concentration (about $0.1 \%$ ) in this serum is identical, or at least close, to the in-vivo sodium concentrations.

Figure 16 shows transverse, coronal and sagittal images of the phantom at both proton and sodium frequencies. Sodium images were acquired with 64 signal averages.

All these results indicate that the performances of the design presented here are good. We think that these performances would be at least as good as, if not better, at the HP ${ }^{129} \mathrm{Xe}$ frequency. Although, the resonance frequency of sodium and xenon are closed, no significant noise from sodium nuclei will disturb the $\mathrm{HP}{ }^{129} \mathrm{Xe}$ signal. Firstly, the excitation plus at $27.7 \mathrm{MHz}$ will not significantly, if never, excite the sodium nuclei. Secondly, the in vivo $\mathrm{HP}{ }^{129} \mathrm{Xe}$ signal would be more than nine or ten orders of magnitude greater than an eventual in vivo sodium signal. Moreover, since the quality factor of the receiving coil is relatively high (about 100), its obtained bandwidth is narrow and it will significantly limit the out-of-band noise. In any case, no influence of the sodium nuclei was observed during our $\mathrm{HP}^{129} \mathrm{Xe}$ NMR experiment.

We are currently building another prototype that includes shielding of the birdcages to minimize external interferences. Some modifications in the mechanical structure of the probe should be achieved to allow for easy delivering of the hyperpolarized xenon to the rat. The next step of our work is the use of this new probe for $r C B F$ in the rat brain. This would be the subject of a future publication. At the same time, a third prototype of the probe is currently being studied for use at a higher magnetic field (4.7 Teslas) for the same application.

Notice finally that the choice of sodium for a first validation of the developed probe is very important, not only because its frequency is close enough to the xenon frequency, but also because of large palette of potential applications of the sodium MRI for both humans and small animals. Actually, MRI is proving to be a very useful tool for sodium quantification in animal models of stroke, ischemia, and cancer in small animals and in humans. For example, ${ }^{23} \mathrm{Na}$ MRI short echo times was used to quantify absolute tissue sodium concentration in patients with brain tumors showing an increase of sodium concentration relative to that in normal brain structures $[14,26]$. In rabbit models of focal cerebral ischemia, it was shown that there are changes in tissue ${ }^{23} \mathrm{Na}$ signal levels following acute ischemia, which may help to identify necrotic tissue and estimate the duration of the ischemia $[27,28]$. Recently, the sodium and proton diffusion MRI are shown to be good biomarkers for early therapeutic response in subcutaneous tumors [26]. Recent advances in ultra-high field MRI hardware have 


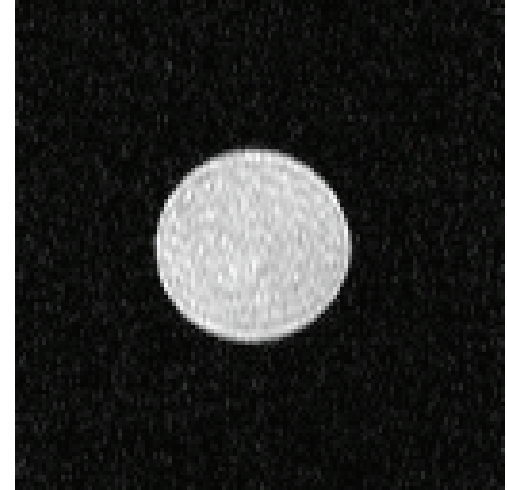

(a)

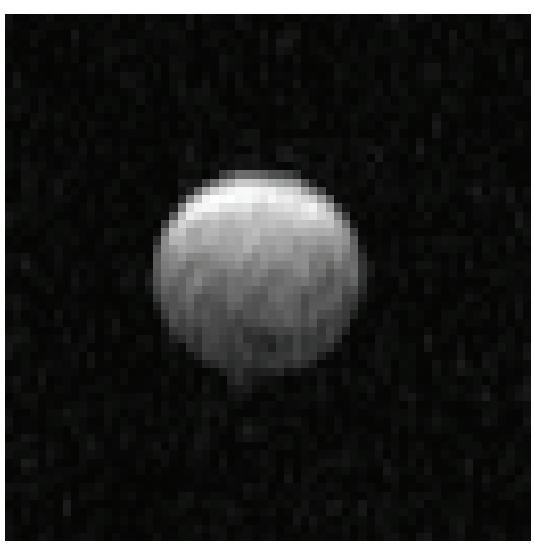

(d)

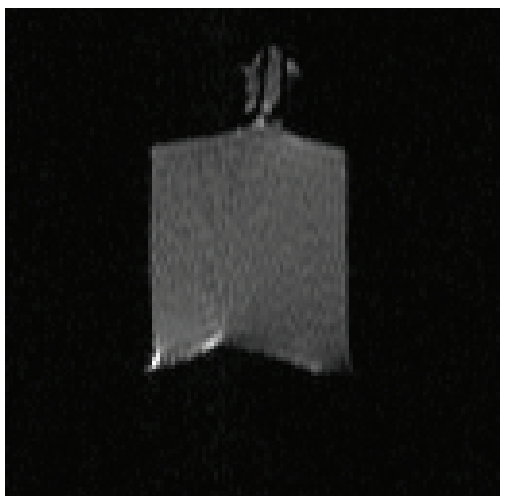

(b)

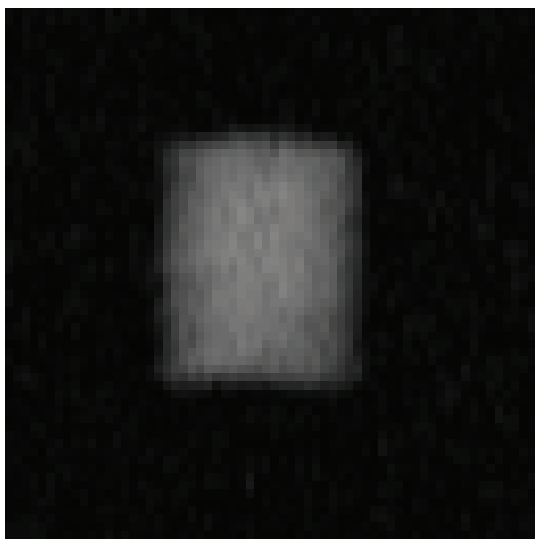

(e)

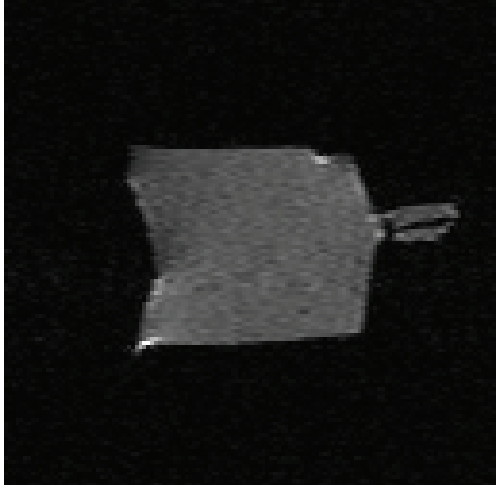

(c)

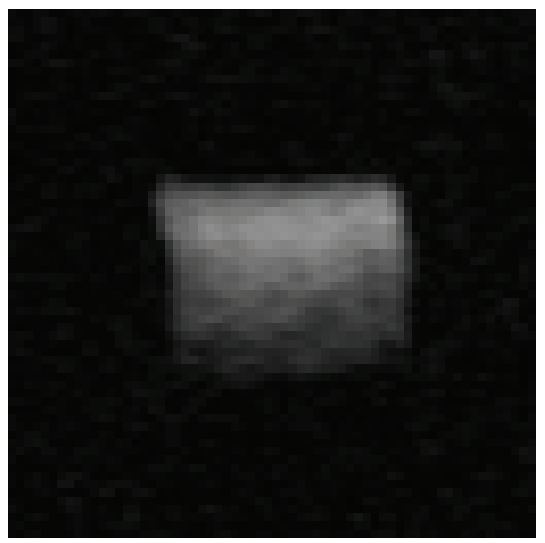

(f)

Figure 16. Cross-sectional, coronal and sagittal MRI images of the syringe (inner diameter=27 $\mathrm{mm}$ ) filled with a physiological serum acquired at (a), (b), (c) proton frequency and at (d), (e), (f) sodium frequency. A spin echo pulse sequence was used to acquire images. FOV (Field of View) $=70 \times 70 \mathrm{~mm}^{2}$, flip angle $=90^{\circ}$, echo time $\mathrm{TE}=17 \mathrm{~ms}$, repetition time $\mathrm{TR}=2000 \mathrm{~ms}$ for proton and $500 \mathrm{~ms}$ for sodium. Slice thickness for proton images $=1 \mathrm{~mm}$. Acquisition matrix was $128 \mathrm{x} 64$ for proton and $64 \mathrm{x} 32$ for sodium. Before transformation of the acquired data to the image space, zero fillings to $128 \times 128$ for proton and to $64 \times 64$ for sodium were applied. Sodium images were acquired with 64 signal averages and without slice selection.

allowed the acquisition of ${ }^{23} \mathrm{Na}$ images of the mouse heart [14]. These examples show that in human and animal models, there is a need to obtain interleaved coregistered anatomical $\left({ }^{1} \mathrm{H}\right)$ and physiological $\left({ }^{23} \mathrm{Na}\right)$ information which may be useful for assessing disease and effective therapeutic intervention. For this purpose, double tuned volume and/or surface coils are required. One of the main advantages of the probe that we presented is the possibility to use it in different configurations according to the application.

Complete schematics of the developed circuits, values and references of all parts, or any other details of design and realization could be obtained by simply writing to the author.

\section{CONCLUSIONS}

In summary, we have reported the design and testing of a new MRI probe consisting of a double-tuned volume coil actively decoupled from a receive-only coil. This developed probe provides proton and sodium images at $2.35 \mathrm{~T}$. In-vivo and phantom ${ }^{1} \mathrm{H}$ images were acquired with the volume transmit/receive coil. ${ }^{23} \mathrm{Na}$ images of phantoms in both transmit/receive mode and in transmitonly/receive-only mode were acquired. In-vivo NMR signals in the rat brain are also obtained. The results showed acceptable performances of the design. This probe is relatively simple to build and it is low-cost. It should be a suitable tool for the HP ${ }^{129} \mathrm{Xe}$ NMR experiments in the rat brain. In its final version, under construction, it should enhance the quantitative measurements of the $r C B F$ and their reliability.

\section{ACKNOWLEDGEMENTS}

The author wishes to thank Christoph Segebarth, Research Director and Director of the Team 5 of GIN (Grenoble-Institute of Neurosciences) and Jean-Louis Leviel for supporting with enthusiasm the research topic on the HP gases in the laboratory. I thank also Olivier Montigon for his help in the realization of the T/R driver circuitry, Vincent Auboiroux for his participation in the mechanical construction 
of the probe. Finally, I am very thankful to Emmanuel Barbier for his support in using his developed MRI sequences and visualization software and for helpful discussions. All these persons are working at the Team 5 of GIN.

\section{REFERENCES}

[1] Albert, M.S. et al. (1994) Biological magnetic resonance imaging using laser polarized ${ }^{129} \mathrm{Xe}$. Nature, 370, 199-201.

[2] Kilian, W. et al. (2004) Dynamic NMR spectroscopy of hyperpolarized ${ }^{129} \mathrm{Xe}$ in human brain analyzed by an uptake model. Magnetic Resonance in Medicine (MRM), 51, 843-847.

[3] Venkatesh, A.K. et al. (2001) Using dynamic hyperpolarized xenon MR to measure brain perfusion. Proceeding of the International Society of Magnetic Resonance in Medicine, 9, 951.

[4] Swanson, S.D. et al. (1997) Brain MRI with laser-polarized ${ }^{129}$ Xe. Magnetic Resonance in Medicine (MRM), 38, 695-698.

[5] Duhamel, G. et al. (2002) Global and regional cerebral blood flow measurements using NMR of injected hyperpolarized xenon-129. Acad Radiol., 9(2), 498-500.

[6] Asfour, A. et al. (2006) Development of a fully digital and low frequency NMR system for polarization measurement of hyperpolarized gases. Proceedings of the IEEE Instrumentation and Measurement Technology Conference IMTC, Sorrento, Italy.

[7] Asfour, A. (2008) A new DAQ-based and versatile low-cost NMR spectrometer working at very-low magnetic field $(4.5 \mathrm{mT})$ : A palette of potential applications. Proceedings of the IEEE International Instrumentation and Measurement Technology Conference $I^{2} M T C$, Vancouver, Canada.

[8] Ackerman, et al. (1980) Mapping of metabolites in whole animals by ${ }^{31} \mathrm{P}$ NMR using surface coils. Nature, 283, 167-170.

[9] Hayes, C.E. et al. (1985) An efficient, highly homogenous radiofrequency coil for whole-body NMR imaging at 1.5 T. Journal of Magnetic Resonance, 63, 622-628.

[10] Fitzsimmons, J.R. et al. (1993) Double Resonant quadrature birdcage. Magnetic Resonance in Medicine (MRM), 30, 107-114.

[11] Rath, A.R. (1990) Design and performance of a double-tuned birdcage coil. Journal of Magnetic Resonance, 86, 488-495.

[12] Shen, G.X. et al. (1997) Dual-frequency, dual-quadrature, birdcage RF coil design with identical $\mathrm{B}_{1}$ pattern for sodium and proton imaging of the human brain at $1.5 \mathrm{~T}$. Magnetic Resonance in Medicine, 38, 717-725.

[13] Lanz, T. et al. (2001) Double tuned ${ }^{23} \mathrm{Na}{ }^{1} \mathrm{H}$ nuclear magnetic resonance birdcage for application on mice in vivo. Review of Scientific Instruments, 72(5), 2508-2510.

[14] Alecci, M. et al. (2006) Practical design of a 4 Tesla double-tuned RF surface coil for interleaved ${ }^{1} \mathrm{H}$ and ${ }^{23} \mathrm{Na}$ MRI of the rat brain. Journal of Magnetic Resonance, 181, 203-211.

[15] Leach, M.O. et al. (1986) The design and use of a dual-frequency surface coil providing proton images for improved localization in ${ }^{31} \mathrm{P}$ spectroscopy of small lesions. Med Phys, 13, 510-513.

[16] Volotovskyy, V. et al. (2002) Doubly tunable double ring surface coil. Concepts in Magnetic Resonance, B(17B), 11-16.

[17] Mispelter, J., Lupu, M. and Briguet, A. (2006) NMR Probeheads for Biophysical and Biomedical Experiments: Theoretical Principles and Practical Guidelines, Imperial College Press.

[18] Chin, C.L. et al. (2002) BirdcageBuilder: Design of specified-geometry birdcage coils with desired current pattern and resonance frequency. Concepts in Magnetic Resonance, 15(2), 156-163.

[19] The ARRL Handbook for Radio Amateurs, Published by the American Radio Relay League, 1996.

[20] Streif, J.U.G. et al. (2003) A coil combination for magnetic resonance perfusion imaging of mice in vivo at $7 \mathrm{~T}$. Review of Scientific Instruments, 74(5), 2843-2848.

[21] Barberi, E.A. et al. (2000) A transmit-only/receive-only (TORO) RF system for high-field MRI/MRS applications. Magnetic Resonance in Medicine (MRM), 43, 284-289.

[22] Décorps, M. et al. (1985) An inductively coupled, series tuned NMR probe. Journal of Magnetic Resonance, $\mathbf{6 5}$, 100-109.

[23] Hoult, D.I. and Lauterburg, P.C. (1979) The sensitivity of the zeugmatographic experiment involving human samples. Journal of Magnetic Resonance, 34, 425-433.

[24] Naritomi, H. et al. (1987) In vivo measurements of the intra- and extra-cellular ${ }^{+} \mathrm{Na}$ and water in the brain and muscle by nuclear magnetic resonance spectroscopy with shift reagent. BIOPHYS Journal, Biophysical Society, 52, 611-616.

[25] Schepkin, V.D. et al. (2006) Sodium and proton diffusion MRI as biomarkers for early therapeutic response in subcutaneous tumors. Magnetic Resonance Imaging, 24, 273-278.

[26] Ouwerkerk, R. et al. (2003) Tissue sodium concentration in human brain tumors as measured with ${ }^{23} \mathrm{Na} \mathrm{MR}$ imaging. Radiology, 227, 529-537.

[27] Bartha, R. et al. (2004) Sodium T2*-weighted MR imaging of acute focal cerebral ischemia in rabbits. Magnetic Resonance Imaging, 22, 983-991.

[28] Wang, Y. et al. (2000) Brain tissue sodium is a ticking clock telling time after arterial occlusion in rat focal cerebral ischemia. Stroke, 31, 1386-1391. 